\title{
Receiver Array Calibration Using Disparate Sources
}

\author{
Ishan Samjeva Daniel Solomon, Member, IEEE, Douglas A. Gray, Member, IEEE, \\ Yuri I. Abramovich, Member, IEEE, and Stuart J. Anderson
}

\begin{abstract}
In this paper, we present a new array calibration procedure for over-the-horizon (OTH) radar, using disparate sources. Unlike previous array calibration methods, which require a specific type or class of sources for calibrating the array, the method we propose can use combinations of singlemode, multimode, and near-field sources; each source with either known or unknown DOA's (directions-of-arrival). Multidimensional MUSIC is exploited for time-invariant DOA sources, while single-snapshot techniques are used for sources that have timevarying DOA's. A nonlinear separable least-squares solution to the array calibration problem is used to estimate the array coupling matrix and sensor positions. Simulation results indicate that good estimates are obtained for the unknown parameters and further the array sidelobe levels and bearing errors are significantly reduced when these estimated parameters are used in array processing. The algorithm performance was also compared with the Cramer-Rao lower bound and found to be statistically efficient.
\end{abstract}

Index Terms - Array calibration, Cramer-Rao lower bound, Jindalee, meteors, OTH radar.

\section{INTRODUCTION}

A RRAY calibration has been an active area of research in array processing for the last few decades with many papers relating mainly to sonar and radar being published in [1]-[29]. For towed sonar hydrophone arrays, receiver gain/phase errors and the time-varying sensor position errors, degrade performance. Radar arrays generally have timeinvariant sensor position errors, but have the additional problem of mutual coupling. In this paper, we consider radar receiver arrays, for example, for bistatic over-the-horizon (OTH) radar applications.

For OTH radar arrays errors in sensor positions, unknown mutual coupling and receiver gain/phase variations are known to degrade performance [30]. Hence, for such radars currently being developed for coastal surveillance, which incorporate antenna arrays that can be erected quickly on unprepared sites, array calibration is essential. While gain/phase errors may be calibrated relatively easily by the injection of signals at the receiver inputs, both sensor position errors and mutual coupling require more sophisticated calibration methods.

Manuscript received September 22, 1997; revised May 26, 1998.

I. S. D. Solomon is with the CRC for Sensor Signal and Information Processing (CSSIP), The Levels, 5095 Australia, and also with the Defence Science and Technology Organization, Salisbury, 5108 Australia.

D. A. Gray is with the University of South Australia, and also with the University of Adelaide, 5000 Australia.

Y. I. Abramovich is with the University of South Australia, The Levels, 5095 Australia.

S. J. Anderson is with the Defence Science and Technology Organization, Salisbury, 5108 Australia.

Publisher Item Identifier S 0018-926X(99)04427-0.

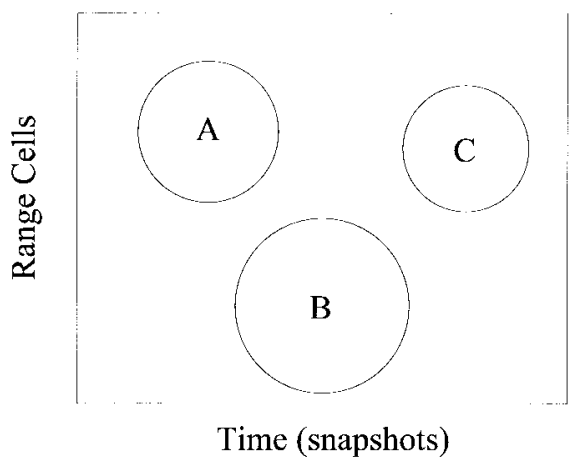

Fig. 1. Illustration of disjoint clusters: $A, B$, and $C$ are disjoint clusters, each of which may contain a number of sources/signals.

For OTH radar array calibration one may use special sources such as beacons, noise sources such as radio stations, and sources of opportunity such as backscattered echoes from meteors. These sources have widely varying properties, which must be accounted for when used for array calibration [31], [32]. For example, in [28], [29] we showed how backscattered echoes from ionized meteor trails may be used for array calibration.

Unlike previous array calibration methods, which require a specific type or class of sources for calibrating the array, the method we propose here can use all available sources for array calibration. For example, the methods proposed by See and $\mathrm{Ng}$ [21]-[23], [27] to estimate sensor positions and mutual coupling, need disjoint single-mode sources of known DOA's and the DOA's must be time-invariant. The method we propose here, however, can use disjoint ${ }^{1}$ clusters (see Fig. 1) of nondisjoint single-mode, multimode, and/or near-field sources, with either known or unknown DOA's. Further, the DOA of each source may be either time-varying or time-invariant.

In Section II, we describe the signal model. In Section III, we consider the case of a cluster of time-varying DOA sources. In Section IV, the case of a cluster of time-invariant DOA sources are considered and we formulate the problem for each case. Then, in Section V, we show how to combine cost functions from a number of clusters of either type into an overall cost function. In Section VI, the proposed algorithm is given with a simulation example in Section VII. Statistical analysis of the algorithm is conducted in Section VIII and the algorithm is compared with the Cramer-Rao lower bound in Section IX. Section X contains the conclusion, while the Appendix give an example and contain mathematical derivations.

\footnotetext{
${ }^{1}$ By disjoint we mean that they do not occupy both the same time snapshots and the same radar range cells.
} 


\section{Signal ModeL}

For a narrowband signal $s_{1}(t)$ impinging an $M$-element array, in the absence of mutual coupling, the output of the $m$ th sensor is

$$
z_{m}(t)=\left(1+\alpha_{m}\right) e^{-j \phi_{m}} s_{1}(t) e^{-j w \tau_{m 1}}+n_{m}(t)
$$

where $\alpha_{m} \& \phi_{m}$ are the gain and phase errors and $n_{m}(t)$ is additive receiver noise. The radar operating radian frequency is $w, \tau_{m n}=\left(x_{m} \sin \theta_{n}+y_{m} \cos \theta_{n}\right) / v, x_{m}$ and $y_{m}$ define the position of the $m$ th sensor for a two-dimensional (2-D) array, $\theta_{1}$ is the $\mathrm{DOA}^{2}$ of the signal (with respect to broadside), and $v$ is the speed of light in free-space.

The vector of $M$ sensor outputs of the array is

$$
\boldsymbol{z}(t)=\boldsymbol{\Gamma} \boldsymbol{a}\left(\theta_{1}\right) s_{1}(t)+n(t)
$$

where $z(t)=\left[z_{1}(t), z_{2}(t), \cdots, z_{M}(t)\right]^{T}, \Gamma=\operatorname{diag}\left\{\left(1+\alpha_{1}\right)\right.$ $\left.e^{-j \phi_{1}}, \cdots,\left(1+\alpha_{M}\right) e^{-j \phi_{M}}\right\}, \boldsymbol{a}\left(\theta_{n}\right)=\left[e^{-j w \tau_{1 n n}}, e^{-j w \tau_{2 n}}\right.$, $\left.\cdots, e^{-j w \tau_{M n}}\right]^{T}, \boldsymbol{n}(t)=\left[n_{1}(t), n_{2}(t), \cdots, n_{M}(t)\right]^{T}$.

In the presence of mutual coupling [33]

$$
\boldsymbol{z}(t)=\boldsymbol{C} \boldsymbol{\Gamma} \boldsymbol{a}\left(\theta_{1}\right) s_{1}(t)+\boldsymbol{n}(t)
$$

where $\boldsymbol{C}=\left(\boldsymbol{I}_{M}+\boldsymbol{Z}_{o} / Z_{L}\right)^{-1}$ is called the coupling matrix. Matrix $\boldsymbol{I}_{M}$ is the $M \times M$ identity matrix, $Z_{O}$ is the array mutual coupling matrix, and $Z_{L}$ is the scalar load impedance. The covariance matrix for this signal, assuming zero mean noise, is

$$
\boldsymbol{R}=E\left\{\boldsymbol{z}(t) z(t)^{H}\right\}
$$

Given either measurements $z(t)$ and/or estimated covariance matrices $\hat{\boldsymbol{R}}$ for a number of signals, the problem is to estimate the sensor positions and coupling matrix by formulating a cost function and minimizing it with respect to these and other unknown parameters. We have assumed here that the data $z(t)$ have been corrected for gain/phase errors.

\section{TIME-VARYING DOA SOURCES}

For time-varying DOA sources one cannot obtain a meaningful spatial covariance matrix due to the nonstationary spatial behavior of the source. Instead, one can use singlesnapshot data for array calibration provided the signal-tonoise ratio (SNR) is sufficiently high. In fact, time-varying DOA sources, which have distinct DOA's each snapshot, can provide multiple single-snapshot sources for array calibration. Examples of time-varying DOA sources are meteor head echoes and calibration sources mounted on a truck or aircraft [31], [32].

For the case of one single-mode source, a single snapshot is

$$
z=\boldsymbol{C a}(\theta) s+n
$$

for which we formulate a nonlinear least squares problem with a cost function

$$
Q=\|z-\boldsymbol{C a}(\theta) s\|^{2} .
$$

\footnotetext{
${ }^{2}$ For time-varying DOA signal $\theta_{1}=\theta_{1}(t)$; however, for simplicity of notation, the dependence is not shown but can be easily inferred from the context.
}

For the case of multiple sources, either uncorrelated or correlated (multimode) signals, which are overlapped in time (nondisjoint), we can generalize (5) as follows for $N$ signals:

$$
z=C A s+n
$$

where $\boldsymbol{A}=\left[\boldsymbol{a}\left(\theta_{1}\right), \boldsymbol{a}\left(\theta_{2}\right), \cdots, \boldsymbol{a}\left(\theta_{N}\right)\right]$, and the vector $\boldsymbol{s}$ of complex signal amplitudes is $\boldsymbol{s}=\left[s_{1}, s_{2}, \cdots, s_{N}\right]^{T}$. The cost function is then

$$
Q=\|z-C A s\|^{2}
$$

\section{TIME-INVARIANT DOA SOURCES}

We call time-invariant DOA sources, those sources whose DOA varies at most marginally from a nominal value. For these sources, one can estimate a spatial covariance matrix. Examples of time-invariant DOA sources are meteor trail echoes and beacon sources [31], [32]. We start this section by outlining the multidimensional MUSIC algorithm and then formulate the problem for time-invariant sources.

Multidimensional MUSIC, proposed originally in [34] (see also [35]), is a multidimensional search procedure for DOA estimation. It does not have the drawback of one-dimensional search procedures (such as MUSIC) of not being able to cope with coherent signals since it does not assume the signal covariance matrix to have full rank. Consider the eigenvectors corresponding to the largest eigenvalues of the covariance matrix (i.e., the signal subspace) and let $\boldsymbol{E}$ be the matrix whose columns are these eigenvectors. It can then be shown [36] that in uncorrelated receiver noise, there exists a matrix/vector $S$ such that

$$
E=C A S
$$

where the elements of $S$ are not necessarily the signal amplitudes (unlike the elements of $s$ in Section III), but rather relate the actual steering vectors (columns of the product $\boldsymbol{C A S}$ ) to the signal eigenvectors in $\boldsymbol{E}$. The category of sources that have to be considered here are:

- one single-mode source;

- one multimode source;

- multiple nondisjoint single-mode sources;

- multiple nondisjoint multimode sources.

We formulate a nonlinear least-squares problem for each cluster of nondisjoint sources as

$$
Q=\|\boldsymbol{E}-\boldsymbol{C A S}\|_{F}^{2}
$$

where $\boldsymbol{E}$ is the $M \times d^{\prime}$ signal subspace matrix/vector, and $S$ is a $d \times d^{\prime}$ matrix/vector for the cluster of nondisjoint sources. (Note the subscript $F$ in (10) denotes that this norm is the Frobenious norm.) The number of signals present is $d$ and the number of signal eigenvalues is $d^{\prime}$. If all the signals are uncorrelated in the cluster then $d^{\prime}=d$ and if any signals are correlated $d^{\prime}<d$.

Note that if a source has a time-invariant DOA for some portion of its duration and has a time-varying DOA for the rest of the time, one can use the approaches in both Sections III and IV for the corresponding time segments. 


\section{Overall Cost Function}

We can combine all subproblems, for each and all of the disjoint clusters, into one problem with a single cost function using the addition property of norms. The cost function is as follows:

$$
Q=\|\tilde{Z}-C A \tilde{S}\|_{F}^{2}
$$

where matrix $\tilde{Z}$ contains a column of $z$ for each different cluster of time-varying DOA sources and column(s) of $\boldsymbol{E}$ for each different cluster of time-invariant sources. $\tilde{S}$ is made up of $S$ 's from each disjoint subproblem/cluster. In the rest of this paper, $N$ will refer to the number of different subproblems/clusters, $N_{T}$ to the total number of signals, and $N_{\tilde{Z}}$ to the number of columns in $\tilde{Z}$. An example of the structure of $\tilde{Z}$ and $\tilde{\boldsymbol{S}}$ is given in the Appendix.

While this problem cannot be solved in general, matrices $\boldsymbol{C}, \boldsymbol{A}$, and $\tilde{S}$ have special structures: the coupling matrix we estimate is symmetric (see [29] for the details), the columns of $\boldsymbol{A}$ are steering vectors, and $\tilde{\boldsymbol{S}}$ is a sparse matrix of known structure. We thus consider minimizing (11) given the special structure of these matrices. Further, the algorithm we propose in the next section is only expected to provide a solution close to the global minimum when the initial parameter values are sufficiently close to the actual parameter values.

Note that for a near-field source, if the range of the source from the radar is known as is the case for backscattered echoes and known transmitters [31], [32], then the steering vector is known except for the DOA and so these sources can be used for array calibration.

\section{AlgORITHM}

The algorithm sequence for this multiparameter optimization was based on the observation that it was possible to significantly improve the sensor position estimates even when the coupling parameters were unknown, but it was not possible to obtain any such improvements in the estimates of the coupling parameters when the sensor position errors were large. By trying to improve the sensor position estimates (at each stage updating the DOA's and complex $s$ 's) and then estimating the coupling matrix (again at each stage updating the DOA's and complex $s$ 's), the cost function is minimized. The algorithm sequence is shown in Fig. 2.

\section{A. Initialization}

As with most nonlinear least-squares problems, the initialization is of paramount importance. First, the nominal sensor position values and the nominal coupling matrix are used with the Bartlett spectrum estimation procedure to obtain initial estimates of each of the unknown signals' DOA's.

For the case of each cluster of time-varying DOA sources the Bartlett spectrum is

$$
p(\theta)=\boldsymbol{v}_{o}(\theta){ }^{H} \boldsymbol{C}_{o}^{H} z_{n} z_{n}^{H} \boldsymbol{C}_{o} \boldsymbol{v}_{o}(\theta)
$$

where $C_{o}$ is the nominal coupling matrix, $\boldsymbol{v}_{o}(\theta)$ is the nominal array steering vector, and $z_{n}$ is the single snapshot of sensor

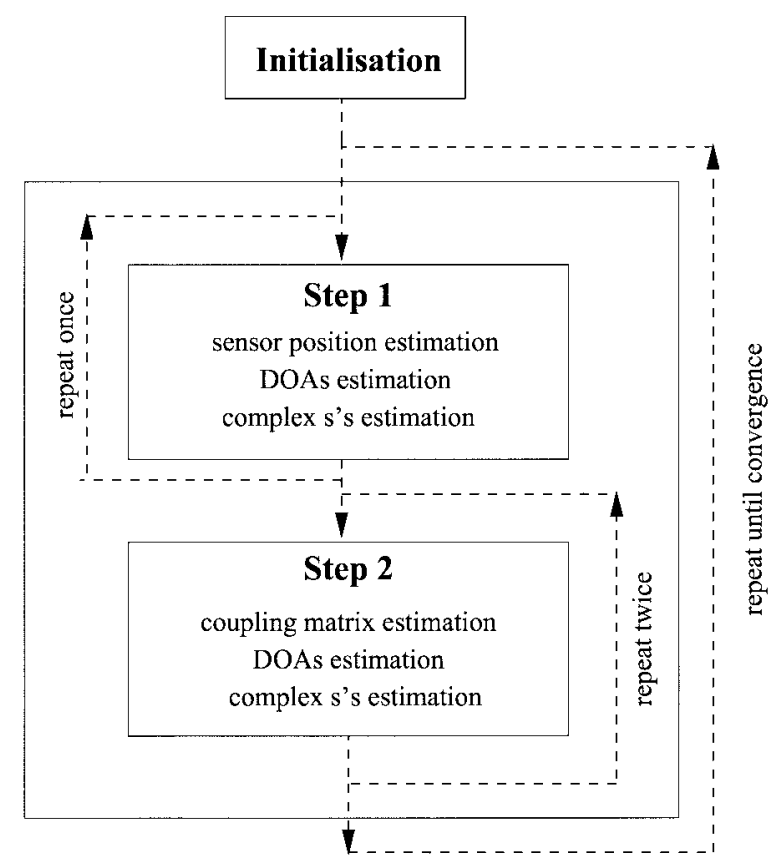

Fig. 2. Algorithm sequence.

outputs for the $n$th cluster of time-varying DOA sources. For the case of each cluster of time-invariant DOA sources

$$
p(\theta)=\boldsymbol{v}_{o}(\theta)^{H} \boldsymbol{C}_{o}^{H} \hat{\boldsymbol{R}}_{n} \boldsymbol{C}_{o} \boldsymbol{v}_{o}(\theta)
$$

where $\hat{\boldsymbol{R}}_{n}$ is the estimated covariance matrix for the $n$th cluster of time-invariant DOA sources.

The peak of each spectra gives the DOA of the strongest source in the cluster. When multiple sources exist (in a cluster) and their DOA's have to be estimated from either a single snapshot or a covariance matrix, the projection matrix is used. For example if for the strongest signal, one obtained a DOA of $\theta_{m}$, then the steering vector for finding the next strongest signal's DOA is

$$
\boldsymbol{v}_{o}^{\prime}(\theta)=\left(\boldsymbol{I}_{M}-\frac{1}{M} \boldsymbol{v}_{o}\left(\theta_{m}\right) \boldsymbol{v}_{o}\left(\theta_{m}\right)^{H}\right) \boldsymbol{v}_{o}(\theta)
$$

This steering vector is then used in the Bartlett spectrum estimator above to obtain the next strongest signal's DOA. This procedure is repeated for obtaining any subsequent signals' DOA's in the cluster.

Once initial values for all these signals' DOA's have been obtained for the cluster, these values together with the nominal sensor positions can be used to form the matrix $A(n)$, of steering vectors for the cluster. Using the nominal coupling matrix and matrix $A(n)$, initial values for the complex $s$ 's for the cluster are estimated as follows for time-varying DOA sources case as

$$
\boldsymbol{s}_{n}=\left(\boldsymbol{C}_{o} \boldsymbol{A}(n)\right)^{+} z_{n}
$$

while for time-invariant DOA sources case

$$
\boldsymbol{S}_{n}=\left(\boldsymbol{C}_{o} \boldsymbol{A}(n)\right)^{+} \boldsymbol{E}_{n}
$$

where ()$^{+}$is the generalized inverse operator. Once all initial complex $s$ 's for all clusters, have been estimated, the matrix $\tilde{S}$ 
in (11) can be formed. Matrix $\boldsymbol{A}$ in (11) is created by simply combining the matrices $A(n)$ columnwise.

\section{B. Sensor Position Estimation}

The last estimates of the DOA's, coupling matrix, and complex $s$ 's are used in this step to estimate the sensor positions. The last estimates of sensor positions are used in a Newton search to update the estimates of the sensor positions.

Let $\boldsymbol{\gamma}(k)=\left[\boldsymbol{x}^{T}(k), \boldsymbol{y}^{T}(k)\right]^{T}$ where $\boldsymbol{x}(k)$ and $\boldsymbol{y}(k)$ are the vectors of estimated sensor positions after the $k$ th iteration. Then

$$
\gamma(k+1)=\boldsymbol{\gamma}(k)-u \boldsymbol{H}^{-1}(\boldsymbol{\gamma}(k)) \boldsymbol{r}(\boldsymbol{\gamma}(k))
$$

where $\boldsymbol{H}$ is the Hessian matrix for the sensor positions, $\boldsymbol{r}$ is the gradient vector for the sensor positions, and the step length is $0<u<1$. Expressions for this Hessian and gradient, which are derived from the cost function in (11), are given in the Appendix.

\section{Coupling Matrix Estimation}

The last estimates of the sensor positions, the signals' DOA's and complex $s$ 's are used in this step to estimate the coupling matrix. It can be shown that the cost function in (11) can be rewritten as follows:

$$
Q=\left\|\tilde{\boldsymbol{z}}^{T}-\boldsymbol{c}^{T} \boldsymbol{F}\right\|^{2}
$$

where $\tilde{\boldsymbol{z}}=\operatorname{vec}(\tilde{\boldsymbol{Z}})$, the $M N_{\tilde{Z}}$ element column vector containing all entries of $\tilde{Z}$ in a column-wise order. Vector $c$ contains the $(M+1) M / 2$ unknown complex coupling matrix parameters since we assume in this paper that the coupling matrix is symmetric (see [29] for the details). Matrix $\boldsymbol{F}$ is created from the matrix product $A \tilde{\boldsymbol{S}}$; an expression for this $(M+1) M / 2 \times M N_{\tilde{Z}}$ matrix is given in the Appendix. The coupling parameters are then estimated as

$$
\boldsymbol{c}=\left(\boldsymbol{F}^{T}\right)^{+} \tilde{z} .
$$

\section{DOA Estimation}

The last estimates of the sensor positions, coupling matrix and complex $s$ 's are used in this step to estimate the signals' DOA's. The last estimate of the signals' DOA's are used in a Newton search to update the estimates of the signals' DOA's. The vector of the signals' DOA's after the $(k+1)$ th iteration is

$$
\boldsymbol{\theta}(k+1)=\boldsymbol{\theta}(k)-u \boldsymbol{H}^{-1}(\boldsymbol{\theta}(k)) \boldsymbol{r}(\boldsymbol{\theta}(k))
$$

where $\boldsymbol{H}$ is the Hessian matrix for the DOA's, $\boldsymbol{r}$ is the gradient vector for the DOA's, and the step length is $0<u<1$. Expressions for this Hessian and gradient, which are derived from the cost function in (11), are given in the Appendix. If any signal has a known DOA, this signal's DOA is not considered in (20).

\section{E. Complex s's Estimation}

The last estimates of the sensor positions, coupling matrix, and the signals' DOA's are used in this step to estimate the complex $s$ 's. For each disjoint cluster of time-varying
DOA sources (15) is used and for each disjoint cluster of time-invariant DOA sources (16) is used.

\section{F. Assumptions}

The following assumptions have been made.

- The number of signals present in each measured snapshot of array outputs and in each estimated covariance matrix is known (see [37] for a method to determine the number of signals). (Note this is not easy in practice when correlated sources exist.)

- A procedure is available for determining whether a source has a time-varying DOA or a time-invariant DOA (see below).

We now suggest a possible approach, which we proposed in [38] for nonstationary detection, to detect variations in the DOA of incident signals. The time interval over which the signal is present is divided into $L$ subintervals of duration $T$. Then $L$ covariance matrices are estimated with the $b$ th covariance matrix (where $b=1,2, \cdots, L$ ) obtained over the interval $(1, b T)$ as follows:

$$
\hat{\boldsymbol{R}}_{b}=\frac{1}{b T} \sum_{t=1}^{b T} z(t) z(t)^{H} .
$$

Note the duration $T$ chosen must be long enough to get a good estimate of $\hat{\boldsymbol{R}}_{1}$ and also short enough so that $\hat{\boldsymbol{R}}_{1}$ represents only information at a particular time.

Eigendecomposing each of the $L$ covariance matrices, one obtains $\hat{\boldsymbol{E}}_{b}$ the signal subspace of $\hat{\boldsymbol{R}}_{b}$ and $\hat{\boldsymbol{U}}_{b}$ the noise subspace of $\hat{\boldsymbol{R}}_{b}$. Using $\hat{\boldsymbol{E}}_{1}$ as a reference, consider the following function:

$$
f(b)=\left\|\hat{\boldsymbol{E}}_{1}^{H} \hat{\boldsymbol{U}}_{b}\right\|^{2}
$$

or alternatively

$$
f(b)=\operatorname{Tr}\left[\hat{\boldsymbol{E}}_{1} \hat{\boldsymbol{E}}_{1}^{H} \hat{\boldsymbol{U}}_{b} \hat{\boldsymbol{U}}_{b}^{H}\right] .
$$

If the signal's DOA is time-invariant, then $f$ should remain small for all $b$, while if the signal's DOA is time-varying $f$ should increase with $b$. Hence, by considering this function it should be possible to detect DOA variations.

Note that since mutual coupling and sensor position errors are both time-invariant over typical radar dwell durations it is possible to conclude any DOA variation detected by the array is due to the received signal rather than the antenna array.

\section{Simulation Example}

Simulations were performed for a 16-element, nominally equispaced linear array with interelement spacing of $d=0.4 \lambda$. The actual sensor positions were $x_{m}=m d+\Delta x_{m}$ and $y_{m}=\Delta y_{m}$, where $\Delta x_{m}$ and $\Delta y_{m}$ are zero mean Gaussian random variables, each with variance of $0.1 d$. The coupling matrix used was an experimentally measured coupling matrix from the Jindalee OTH radar transmitting array (situated in central Australia). The initial sensor positions were taken to be the nominal sensor positions, while the identity matrix was 

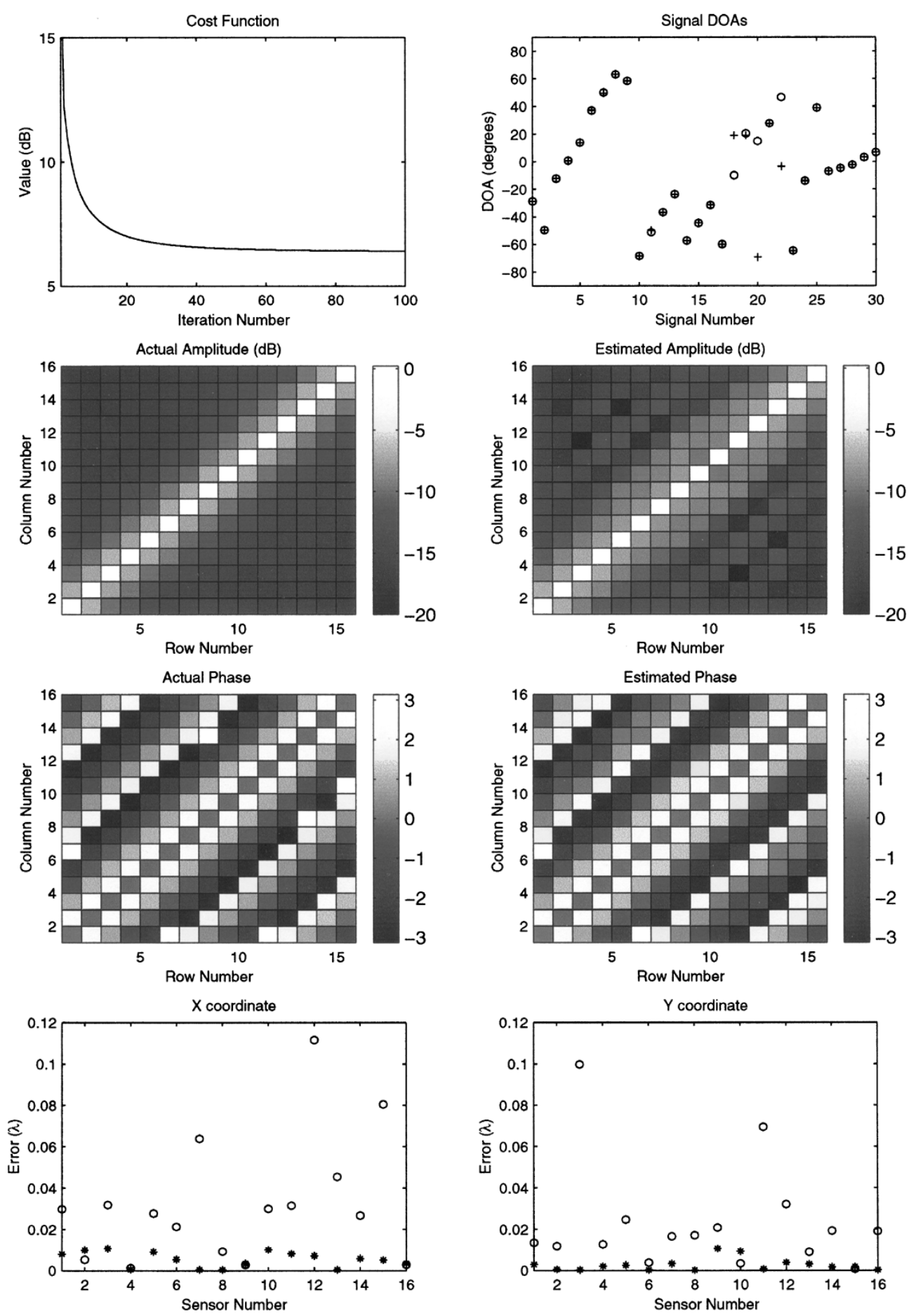

Fig. 3. Simulation example results: cost function decreases with iteration number; the actual (o) and the estimated (+) signals' DOAs; actual coupling matrix and estimated coupling matrix; errors in nominal $(0)$ and final $(*)$ sensor position estimates.

used as the initial coupling matrix. The termination criterion was for the algorithm to stop when the cost function $Q$ changed in value by less than $0.1 \%$.

In total, 30 sources were used with SNR's of $30 \mathrm{~dB}$. The number of snapshots obtained from the time-invariant DOA sources was 500. Disjoint clusters of time-varying DOA sources: single source (two clusters), three sources (two clusters), single known DOA source (several clusters). Disjoint clusters of time-invariant DOA sources: single source (two clusters), three correlated sources (two clusters), three uncorrelated sources (one cluster), two correlated sources with a single uncorrelated source (one cluster).

The results obtained are displayed in Fig. 3.

- The cost function initially decreases rapidly and then tends to level off after about 25 iterations.

- The signals' DOA's estimates are good in most cases (the largest DOA errors seem to be associated with clusters that have multiple signal eigenvectors). 

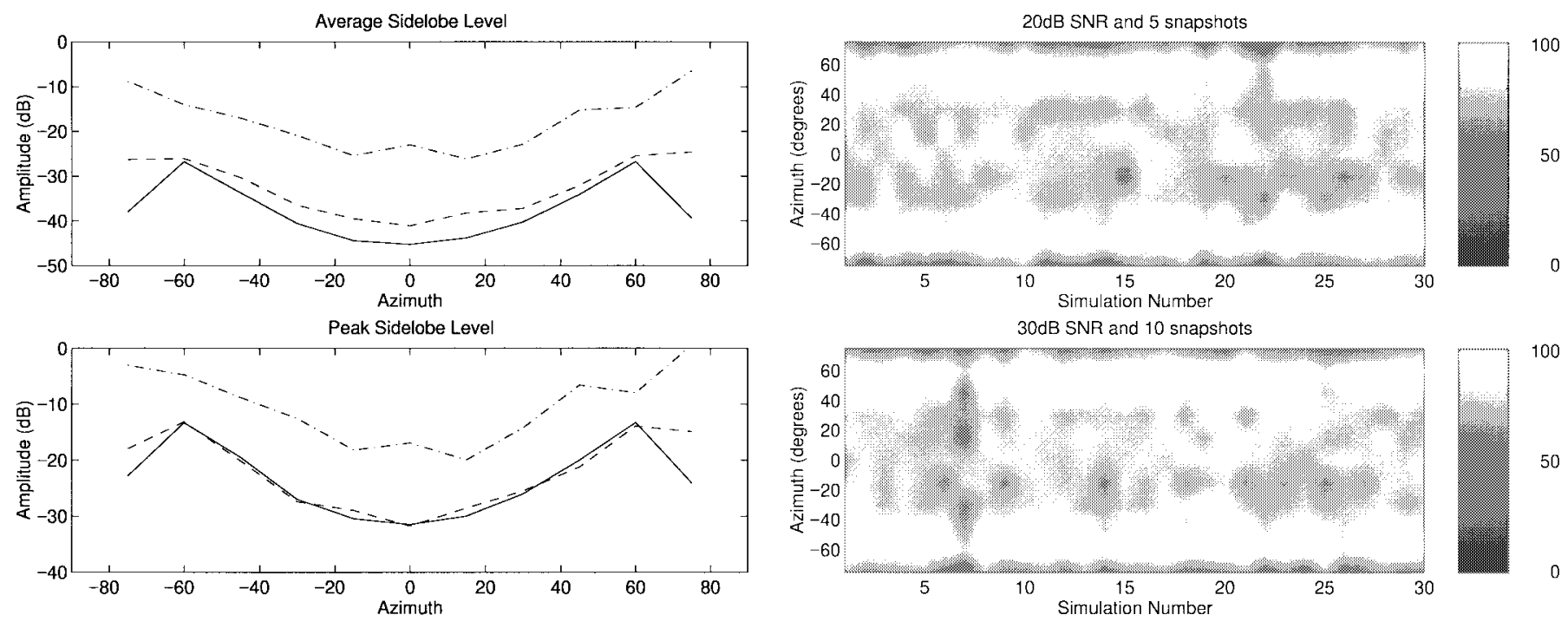

Fig. 4. Average and peak sidelobe levels for the nominal parameters $(-\cdot-)$, estimated parameters $(--)$ and also the actual parameters $(-)$.

- The magnitude and phase of the coupling matrix used and the coupling matrix estimated are in close agreement (the phase is unwrapped for easy comparison).

- The errors in the $x$ and $y$ coordinates after the final iteration $(*)$ are much smaller than the errors in the nominal values (o).

The degradation due to uncompensated mutual coupling and sensor position errors on radar performance has been quantified in [30]; the main degradation was found to be in the array sidelobe levels and it was shown that this resulted in poor performance in interference environments. Hence, to analyze the performance of the algorithm, one needs to compare the sidelobe levels obtained using the estimated parameters with those of the uncalibrated array. It can be shown that given the sensor positions and the coupling matrix, the weighting required to obtain minimum average sidelobes is

$$
\boldsymbol{w}\left(\theta_{o}\right)=\frac{\left(\hat{\boldsymbol{C}}^{H}\right)^{-1} D^{-1} \hat{\boldsymbol{a}}\left(\theta_{o}\right)}{\hat{\boldsymbol{a}}\left(\theta_{o}\right)^{H} \boldsymbol{D}^{-1} \hat{\boldsymbol{a}}\left(\theta_{o}\right)}
$$

where $\hat{C}$ is the estimated coupling matrix and $\hat{\boldsymbol{a}}(\phi)$ is the steering vector formed using the estimated sensor positions. The matrix $\boldsymbol{D}=\int \hat{\boldsymbol{a}}(\phi) \hat{\boldsymbol{a}}(\phi)^{H} \cos (\phi) d \phi$, where the integral is over the sidelobe region. The array beam pattern is then

$$
B_{\theta_{o}}(\phi)=\left\|\boldsymbol{w}\left(\theta_{o}\right)^{H} \boldsymbol{v}(\phi)\right\|^{2}
$$

where $\theta_{0}$ is the steer direction of the beam, $\phi$ is the DOA considered, and $\boldsymbol{v}(\phi)$ is the actual steering vector (including the effects of mutual coupling). Using (25) the sidelobe levels can then be calculated.

In order to obtain the sidelobe levels using the parameters estimated in Fig. 3, the sidelobe region required to compute matrix $D$ was chosen so that the resulting beam had the same beamwidth as the Hamming window. Fig. 4 shows the sidelobe levels for this example where both the average and peak sidelobe levels are shown as a function of azimuth. The sidelobe levels for the estimated parameters $(--)$ is much

Fig. 5. Performance of algorithm for typical number of snapshots and SNR obtainable from OTH radar sources.

better than the sidelobe levels for the nominal parameters $(-$. -) and is close to the sidelobe levels for the actual parameters $(-)$. Hence, we conclude the algorithm has performed well. It was also observed that the bearing errors were reduced.

\section{Statistical AnAlysis}

A statistical analysis is required to analyze the algorithm's performance in detail. The parameter we consider here is

$$
\eta(\theta)=\frac{S L B_{\mathrm{nom}}(\theta)-S L B_{\mathrm{est}}(\theta)}{S L B_{\mathrm{nom}}(\theta)-S L B_{\mathrm{act}}(\theta)} * 100
$$

where $S L B_{\text {nom }}(\theta), S L B_{\text {est }}(\theta), S L B_{\text {act }}(\theta)$ are the average sidelobe level obtained with nominal parameters, with estimated parameters and with actual parameters respectively. Each quantity is expressed in decibels and as a function of bearing $\theta$. Hence, if $\eta(\theta)$ is small (close to zero) the algorithm has not performed well, while if $\eta(\theta)$ is large (close to hundred) the algorithm has performed well.

We initially conducted 100 simulations with the sensor position errors being randomly generated in each case and observed that the algorithm performed very well in all cases, with no failures. To test the algorithm performance for typical SNR and number of snapshots obtainable from OTH radar sources, simulations were conducted for different SNRsnapshot combinations. Two combinations are shown in Fig. 5, where the SNR and number of snapshots are $20 \mathrm{~dB}$ and 5, respectively, in the top image and $30 \mathrm{~dB}$ and 10 in the bottom image. Note, however, the SNR of time-varying DOA clusters (single snapshot sources) are $30 \mathrm{~dB}$ in both cases. The same coupling matrix was used in all the simulations.

Clearly, the algorithm has performed very well, robust results being obtained even for a few snapshots. We have, however, observed that poor results are obtained when the SNR of time-varying DOA clusters (single snapshot sources) is not high (below $25 \mathrm{~dB}$ ), as one would expect.

The algorithm's performance can be further improved by using better initial values for the parameters being estimated. 

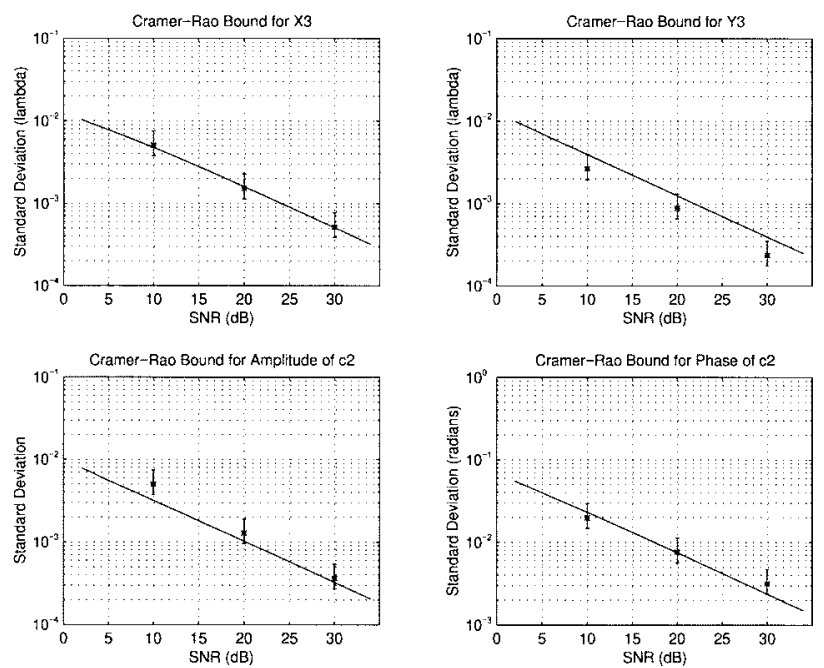

Fig. 6. Algorithm performance relative to theoretical performance bound: STD (*) and $99 \%$ confidence intervals (vertical bar) as a function of SNR for the position of a sensor and for the amplitude and phase of a coupling parameter. The solid line is the CRLB. Thirty algorithm runs were used to obtain each STD value.

Note that for the coupling matrix, instead of the identity matrix, which has been used for these simulations as the initial value, any modeled or previously estimated coupling matrix could be used. While, in general, we cannot guarantee convergence to the global minimum, the results obtained indicate that good results are obtained for typical initial conditions.

\section{CRAmer-RAO BOUnd}

The algorithm's performance was compared to the Cramer-Rao lower bound (CRLB) for clusters of multiple snapshot time-invariant DOA sources. It was assumed that the location of one sensor and the direction to another is known and, hence, for the four-element array considered, there were only five sensor positions $(2 M-3)$ unknown. Since we assume a symmetric coupling and placed the constraint that $C_{11}=1,18$ coupling values $(M(M+1)-2)$ are unknown. We considered 11 disjoint clusters, each with a single-mode source and another disjoint cluster with a single multimode source (with three correlated signals), all signals with unknown DOA's; hence, 14 DOA's $\left(N_{T}\right)$ are unknown. The total number of unknown parameters is, therefore, 37 (the noise power is assumed to be known). The CRLB is derived in [36].

The solid line in Fig. 6 is the CRLB, where 500 snapshots are obtained from these time-invariant DOA sources. We have over-plotted the standard deviation (STD) values $(*)$ estimated from 30 algorithm runs and the $99 \%$ confidence intervals (vertical bar). The results clearly show that the algorithm attains the CRLB and is hence statistically efficient. We obtained similar results for the other unknown parameters and in each case the bias was small compared to the STD.

When multiple snapshot time-invariant DOA sources and single-snapshot time-varying DOA sources were used together, we observed that the algorithm's performance departed from the corresponding CRLB. This somewhat expected result is due to only single snapshots being obtained from the time-varying DOA sources; the CRLB being only achieved asymptotically (i.e., large number of snapshots and SNR).

\section{CONCLusion}

A new array calibration approach, which can combine signals from disparate sources to estimate mutual coupling and sensor position errors, has been presented. The performance of the algorithm has been analyzed using simulations, illustrating that the algorithm significantly reduces the array sidelobes and bearing errors. It was also shown that the algorithm performed well for typical SNR and number of snapshots obtainable from OTH radar sources and that the algorithm is statistically efficient. In general, while convergence to the global minimum cannot be guaranteed for this algorithm, good results are obtained for typically available initial conditions.

\section{APPENDIX \\ AN EXAMPLE}

Consider disjoint clusters of time-varying DOA sources: one cluster of a single source; one cluster of three sources (either correlated or uncorrelated); disjoint clusters of time-invariant DOA sources: one cluster of a single source; one cluster of three uncorrelated sources. For this case, $N=4, N_{T}=8$, the matrix $\tilde{\boldsymbol{Z}}(M \times 6)$ and $\tilde{\boldsymbol{S}}(8 \times 6)$ are as given below, where $\boldsymbol{z}_{1}, \boldsymbol{z}_{2}$ are vectors of single snapshots and $\boldsymbol{e}_{1}, \boldsymbol{E}_{2}$ is a vector and a matrix of eigenvectors

$$
\begin{aligned}
\tilde{\boldsymbol{Z}}= & {\left[\boldsymbol{z}_{1}, \boldsymbol{z}_{2}, \boldsymbol{e}_{1}, \boldsymbol{E}_{2}\right] } \\
\tilde{\boldsymbol{S}} & =\left[\begin{array}{cccccc}
s_{11} & 0 & 0 & 0 & 0 & 0 \\
0 & s_{21} & 0 & 0 & 0 & 0 \\
0 & s_{22} & 0 & 0 & 0 & 0 \\
0 & s_{23} & 0 & 0 & 0 & 0 \\
0 & 0 & s_{31} & 0 & 0 & 0 \\
0 & 0 & 0 & s_{41} & s_{44} & s_{47} \\
0 & 0 & 0 & s_{42} & s_{45} & s_{48} \\
0 & 0 & 0 & s_{43} & s_{46} & s_{49}
\end{array}\right] .
\end{aligned}
$$

\section{Algorithm Details}

A. Expression for matrix $F$ in (18): It can be shown that for the symmetric coupling matrix we employ, the matrix $\boldsymbol{F}$ in (18) is given for all $1 \leq m \leq M$ and $1 \leq n \leq N_{\tilde{Z}}$ as

$$
\begin{aligned}
& F_{l,(n-1) M+m} \\
& \quad= \begin{cases}T_{m+l-\rho(m), n} & \rho(m) \leq l \leq \rho(m+1)-1 \\
T_{h, n} & l=\rho(s)+m-h \quad \forall 1 \leq h<m-1\end{cases}
\end{aligned}
$$

where $T=A \tilde{S}$, and

$$
\rho(m)= \begin{cases}1 & m=1 \\ \rho(m-1)+(M-m+2) & 2 \leq m \leq M \\ \rho(m-1)+1 & m=M+1\end{cases}
$$


B. Gradient and Hessian in (17): The $2 M \times 1$ gradient vector is $\boldsymbol{r}(\gamma(k))=\left[(\partial Q / \partial \boldsymbol{x}(k))^{T},(\partial Q / \partial \boldsymbol{y}(k))^{T}\right]^{T}$, and the $2 M \times 2 M$ Hessian matrix is

$$
\boldsymbol{H}(\gamma(k))=\left[\begin{array}{ll}
\partial^{2} Q / \partial \boldsymbol{x}(k) \partial \boldsymbol{x}^{T}(k) & \partial^{2} Q / \partial \boldsymbol{x}(k) \partial \boldsymbol{y}^{T}(k) \\
\partial^{2} Q / \partial \boldsymbol{y}(k) \partial \boldsymbol{x}^{T}(k) & \partial^{2} Q / \partial \boldsymbol{y}(k) \partial \boldsymbol{y}^{T}(k)
\end{array}\right]
$$

One can rewrite $Q$ in (11) as

$$
\begin{aligned}
Q \approx & \sum_{l=1}^{L}\left\|\tilde{z}_{l}-\boldsymbol{C} \boldsymbol{A} \tilde{\boldsymbol{s}}_{l}\right\|^{2} \\
= & \sum_{l=1}^{L}\left(\tilde{z}_{l}^{H} \tilde{z}_{l}-\tilde{z}_{l}^{H} \boldsymbol{C A} \tilde{\boldsymbol{s}}_{l}-\tilde{\boldsymbol{s}}_{l}^{H} \boldsymbol{A}^{H} \boldsymbol{C}^{H} \tilde{z}_{l}\right. \\
& \left.+\tilde{\boldsymbol{s}}_{l}^{H} \boldsymbol{A}^{H} \boldsymbol{C}^{H} \boldsymbol{C} \boldsymbol{A} \tilde{\boldsymbol{s}}_{l}\right)
\end{aligned}
$$

where $\tilde{z}_{l}$ is the $l$ th (of $L$ ) column of $\tilde{Z}$ and $\tilde{\boldsymbol{s}}_{l}$ is the $l$ th column of $\tilde{S}$.

Now define $\dot{\boldsymbol{A}}_{x_{p}}=\partial \boldsymbol{A} / \partial x_{p}=\boldsymbol{D} \boldsymbol{x}_{\boldsymbol{p}} \odot \boldsymbol{A}$, where $\odot$ is the Hadamard product and $\boldsymbol{D} \boldsymbol{x}_{\boldsymbol{p}}$ is an $M \times N_{T}$ matrix with all but the $p$ th row zero; the $n$th element of the $p$ th row is $(-2 \pi j / \lambda) \sin \left(\theta_{n}\right)$, where $\lambda$ is the radar wavelength. Then

$$
\begin{aligned}
\partial Q / \partial x_{p}= & \sum_{l=1}^{L}\left(-\tilde{z}_{l}^{H} \boldsymbol{C} \dot{\boldsymbol{A}}_{x_{p}} \tilde{\boldsymbol{s}}_{l}-\tilde{\boldsymbol{s}}_{l}^{H} \dot{\boldsymbol{A}}_{x_{p}}^{H} \boldsymbol{C}^{H} \tilde{z}_{l}\right. \\
& \left.+\tilde{\boldsymbol{s}}_{l}^{H} \dot{\boldsymbol{A}}_{x_{p}}^{H} \boldsymbol{C}^{H} \boldsymbol{C} \boldsymbol{A} \tilde{\boldsymbol{s}}_{l}+\tilde{\boldsymbol{s}}_{l}^{H} \boldsymbol{A}^{H} \boldsymbol{C}^{H} \boldsymbol{C} \dot{\boldsymbol{A}}_{x_{p}} \tilde{\boldsymbol{s}}_{l}\right) \\
= & -2 \Re\left\{\sum_{l=1}^{L}\left(\tilde{z}_{l}^{H}-\tilde{\boldsymbol{s}}_{l}^{H} \boldsymbol{A}^{H} \boldsymbol{C}^{H}\right) \boldsymbol{C}\left(\boldsymbol{D} \boldsymbol{x}_{\boldsymbol{p}} \odot A\right) \tilde{\boldsymbol{s}}_{l}\right\}
\end{aligned}
$$

and hence

$$
\begin{aligned}
\partial^{2} Q / \partial^{2} x_{p} & \\
=-2 \Re\{ & \left\{\sum _ { l = 1 } ^ { L } \left[\left(\tilde{\boldsymbol{z}}_{l}^{H}-\tilde{\boldsymbol{s}}_{l}^{H} \boldsymbol{A}^{H} \boldsymbol{C}^{H}\right) \boldsymbol{C}\left(\boldsymbol{D} \boldsymbol{x}_{\boldsymbol{p}} \odot \boldsymbol{D} \boldsymbol{x}_{\boldsymbol{p}} \odot \boldsymbol{A}\right) \tilde{\boldsymbol{s}}_{l}\right.\right. \\
& \left.\left.-\tilde{\boldsymbol{s}}_{l}^{H}\left(A^{H} \odot \boldsymbol{D} \boldsymbol{x}_{\boldsymbol{p}}^{H}\right) \boldsymbol{C}^{H} \boldsymbol{C}\left(\boldsymbol{D} \boldsymbol{x}_{\boldsymbol{p}} \odot \boldsymbol{A}\right) \tilde{\boldsymbol{s}}_{l}\right]\right\}
\end{aligned}
$$

$$
\begin{gathered}
\partial^{2} Q / \partial x_{p} \partial x_{q} \\
=-2 \Re\left\{\sum _ { l = 1 } ^ { L } \left[\left(\tilde{\boldsymbol{z}}_{l}^{H}-\tilde{\boldsymbol{s}}_{l}^{H} A^{H} \boldsymbol{C}^{H}\right) \boldsymbol{C}\left(\boldsymbol{D} \boldsymbol{x}_{\boldsymbol{p}} \odot \boldsymbol{D} \boldsymbol{x}_{\boldsymbol{q}} \odot \boldsymbol{A}\right) \tilde{\boldsymbol{s}}_{l}\right.\right. \\
\left.\left.\quad-\tilde{\boldsymbol{s}}_{l}^{H}\left(\boldsymbol{A}^{H} \odot \boldsymbol{D} \boldsymbol{x}_{\boldsymbol{q}}^{H}\right) \boldsymbol{C}^{H} \boldsymbol{C}\left(\boldsymbol{D} \boldsymbol{x}_{\boldsymbol{p}} \odot A\right) \tilde{\boldsymbol{s}}_{l}\right]\right\} \\
=2 \Re\left\{\sum_{l=1}^{L} \tilde{\boldsymbol{s}}_{l}^{H}\left(\boldsymbol{A}^{H} \odot \boldsymbol{D} \boldsymbol{x}_{\boldsymbol{q}}^{H}\right) \boldsymbol{C}^{H} \boldsymbol{C}\left(\boldsymbol{D} \boldsymbol{x}_{\boldsymbol{p}} \odot A\right) \tilde{\boldsymbol{s}}_{l}\right\}
\end{gathered}
$$

where $\Re\{\}$ is the real part, $q \neq p$, and $\boldsymbol{D} \boldsymbol{x}_{\boldsymbol{q}}$ is an $M \times N_{T}$ element matrix with all but the $q$ th row zero; the $n$th element of the $q$ th row is $(-2 \pi j / \lambda) \sin \left(\theta_{n}\right)$. Equation (35) is obtained from (34) since $\boldsymbol{D} \boldsymbol{x}_{\boldsymbol{p}} \odot \boldsymbol{D} \boldsymbol{x}_{\boldsymbol{q}}$ is the zero matrix for $q \neq p$. The partial derivatives with respect to the $y$ coordinates are obtained by replacing $\boldsymbol{D} \boldsymbol{x}_{\boldsymbol{p}}$ by $\boldsymbol{D} \boldsymbol{y}_{\boldsymbol{p}}$, where $\boldsymbol{D} \boldsymbol{y}_{\boldsymbol{p}}$ is again an $M \times N_{T}$ element matrix with all but the $p$ th row zero; the $n$th element of the $p$ th row is $(-2 \pi j / \lambda) \cos \left(\theta_{n}\right)$ in this case. The $\partial^{2} Q / \partial x_{p} \partial y_{q}$ terms are similarly obtained (see [36]).

C. Gradient and Hessian in (20): The $N_{T} \times 1$ gradient vector is $\boldsymbol{r}(\boldsymbol{\theta}(k))=\partial Q / \partial \boldsymbol{\theta}(k)$ and the $N_{T} \times N_{T}$ Hessian matrix is $\boldsymbol{H}(\boldsymbol{\theta}(k))=\partial^{2} Q / \partial \boldsymbol{\theta}(k) \partial \boldsymbol{\theta}^{T}(k)$.

Now define $\dot{A}_{\theta_{p}}=\partial A / \partial \theta_{p}=D\left(\theta_{p}\right) A \boldsymbol{e}_{p} \boldsymbol{e}_{p}^{T}$, where $D\left(\theta_{p}\right)=(-2 \pi j / \lambda) \operatorname{diag}\left\{\boldsymbol{x} \cos \left(\theta_{p}\right)-\boldsymbol{y} \sin \left(\theta_{p}\right)\right\}$, and $e_{p}$ is the unit vector with all but the $p$ th element zero.

Then from (31)

$$
\begin{aligned}
\partial Q / \partial \theta_{p}= & \sum_{l \in L(p)}\left(-\tilde{z}_{l}^{H} \boldsymbol{C} \dot{\boldsymbol{A}}_{\theta_{p}} \tilde{\boldsymbol{s}}_{l}-\tilde{\boldsymbol{s}}_{l}^{H} \dot{\boldsymbol{A}}_{\theta_{p}}^{H} C^{H} \tilde{\boldsymbol{z}}_{l}\right. \\
& \left.+\tilde{\boldsymbol{s}}_{l}^{H} \dot{\boldsymbol{A}}_{\theta_{p}}^{H} \boldsymbol{C}^{H} \boldsymbol{C} \boldsymbol{A} \tilde{\boldsymbol{s}}_{l}+\tilde{\boldsymbol{s}}_{l}^{H} \boldsymbol{A}^{H} \boldsymbol{C}^{H} \boldsymbol{C} \dot{\boldsymbol{A}}_{\theta_{p}} \tilde{\boldsymbol{s}}_{l}\right) \\
= & -2 \Re\left\{\sum _ { l \in L ( p ) } \left[\tilde{\boldsymbol{z}}_{l}^{H} \boldsymbol{C D}\left(\theta_{p}\right) \boldsymbol{A} \boldsymbol{e}_{p} \boldsymbol{e}_{p}^{T} \tilde{\boldsymbol{s}}_{l}\right.\right. \\
& \left.\left.-\tilde{\boldsymbol{s}}_{l}^{H} \boldsymbol{A}^{H} \boldsymbol{C}^{H} \boldsymbol{C D}\left(\theta_{p}\right) A \boldsymbol{e}_{p} \boldsymbol{e}_{p}^{T} \tilde{\boldsymbol{s}}_{l}\right]\right\} \\
= & -2 \Re\left\{\sum_{l \in L(p)}\left(\tilde{z}_{l}^{H}-\tilde{\boldsymbol{s}}_{l}^{H} \boldsymbol{A}^{H} \boldsymbol{C}^{H}\right) \boldsymbol{C D}\left(\theta_{p}\right) \boldsymbol{A} \boldsymbol{e}_{p} \boldsymbol{e}_{p}^{T} \tilde{\boldsymbol{s}}_{l}\right\}
\end{aligned}
$$

where $L(p)$ is the set of all $l$ indexes corresponding to the cluster containing $\theta_{p}$.

From (36)

$$
\begin{aligned}
& \partial^{2} Q / \partial^{2} \theta_{p}=-2 \Re\left\{\sum _ { l \in L ( p ) } \left[\tilde{z}_{l}^{H} \boldsymbol{C} \dot{\boldsymbol{D}}_{\theta_{p}}\left(\theta_{p}\right) \boldsymbol{A} \boldsymbol{e}_{p} \boldsymbol{e}_{p}^{T} \tilde{\boldsymbol{s}}_{l}\right.\right. \\
& +\tilde{\boldsymbol{z}}_{l}^{H} \boldsymbol{C D}\left(\theta_{p}\right) \dot{A}_{\theta_{p}} \boldsymbol{e}_{p} \boldsymbol{e}_{p}^{T} \tilde{\boldsymbol{s}}_{l} \\
& -\tilde{\boldsymbol{s}}_{l}^{H} \dot{\boldsymbol{A}}_{\theta_{p}}^{H} \boldsymbol{C}^{H} \boldsymbol{C D}\left(\theta_{p}\right) \boldsymbol{A} \boldsymbol{e}_{p} \boldsymbol{e}_{p}^{T} \tilde{\boldsymbol{s}}_{l} \\
& -\tilde{\boldsymbol{s}}_{l}^{H} \boldsymbol{A}^{H} \boldsymbol{C}^{H} \boldsymbol{C} \dot{\boldsymbol{D}}_{\theta_{p}}\left(\theta_{p}\right) \boldsymbol{A} \boldsymbol{e}_{p} \boldsymbol{e}_{p}^{T} \tilde{\boldsymbol{s}}_{l} \\
& \left.\left.-\tilde{\boldsymbol{s}}_{l}^{H} \boldsymbol{A}^{H} \boldsymbol{C}^{H} \boldsymbol{C D}\left(\theta_{p}\right) \dot{\boldsymbol{A}}_{\theta_{p}} \boldsymbol{e}_{p} \boldsymbol{e}_{p}^{T} \widetilde{\boldsymbol{s}}_{l}\right]\right\}
\end{aligned}
$$

where $\dot{\boldsymbol{D}}_{\theta_{p}}=\partial \boldsymbol{D} / \partial \theta_{p}=\boldsymbol{D}_{2}\left(\theta_{p}\right)=(2 \pi j / \lambda) \operatorname{diag}\left\{\boldsymbol{x} \sin \left(\theta_{p}\right)\right.$ $\left.+\boldsymbol{y} \cos \left(\theta_{p}\right)\right\}$. Hence

$$
\begin{aligned}
& \partial^{2} Q / \partial^{2} \theta_{p}=-2 \Re\left\{\sum _ { l \in L ( p ) } \left[\tilde { \boldsymbol { z } } _ { l } ^ { H } \boldsymbol { C } \left(\boldsymbol{D}_{2}\left(\theta_{p}\right)\right.\right.\right. \\
& \left.+\boldsymbol{D}\left(\theta_{p}\right) \boldsymbol{D}\left(\theta_{p}\right)\right) \boldsymbol{A} \boldsymbol{e}_{p} \boldsymbol{e}_{p}^{T} \tilde{\boldsymbol{s}}_{l} \\
& +\left(\tilde{\boldsymbol{s}}_{l}^{H} \boldsymbol{e}_{p} \boldsymbol{e}_{p}^{T} \boldsymbol{A}^{H} \boldsymbol{D}\left(\theta_{p}\right) \boldsymbol{C}^{H} \boldsymbol{C D}\left(\theta_{p}\right)\right. \\
& \text { - } \tilde{\boldsymbol{s}}_{l}^{H} A^{H} C^{H} \boldsymbol{C}\left(\boldsymbol{D}_{2}\left(\theta_{p}\right)\right. \\
& \left.\left.\left.\left.+\boldsymbol{D}\left(\theta_{p}\right) \boldsymbol{D}\left(\theta_{p}\right)\right)\right) A \boldsymbol{e}_{p} \boldsymbol{e}_{p}^{T} \tilde{\boldsymbol{s}}_{l}\right]\right\} \text {. }
\end{aligned}
$$


Now from (37)

$$
\begin{aligned}
\partial^{2} Q / \partial \theta_{p} \partial \theta_{q} & =+2 \Re\left\{\sum_{l \in L(p)} \tilde{\boldsymbol{s}}_{l}^{H} \dot{A}_{\theta_{q}}^{H} \boldsymbol{C}^{H} \boldsymbol{C D}\left(\theta_{p}\right) A \boldsymbol{e}_{p} \boldsymbol{e}_{p}^{T} \tilde{\boldsymbol{s}}_{l}\right\} \\
& =-2 \Re\left\{\sum_{l \in L(p)} \tilde{\boldsymbol{s}}_{l}^{H} \boldsymbol{e}_{q} \boldsymbol{e}_{q}^{T} \boldsymbol{A}^{H} \boldsymbol{D}\left(\theta_{q}\right) \boldsymbol{C}^{H} \boldsymbol{C D}\left(\theta_{p}\right) A \boldsymbol{e}_{p} \boldsymbol{e}_{p}^{T} \tilde{\boldsymbol{s}}_{l}\right\}
\end{aligned}
$$

where $q \neq p$ and $\boldsymbol{D}\left(\theta_{q}\right)=(-2 \pi j / \lambda) \operatorname{diag}\left\{\boldsymbol{x} \cos \left(\theta_{q}\right)-\right.$ $\left.y \sin \left(\theta_{q}\right)\right\}$.

\section{REFERENCES}

[1] C. N. Dorny, "A self-survey technique for self-cohering of antenna systems," IEEE Trans. Antennas Propagat., vol. AP-26, pp. 877-881, Nov. 1978.

[2] C. N. Dorny and B. S. Meagher Jr., "Cohering of an experimental nonrigid array by self-survey," IEEE Trans. Antennas Propagat., vol. AP-28, pp. 902-904, Nov. 1980.

[3] B. D. Steinberg, Microwave Imaging with Large Antenna Arrays. New York: Wiley, 1983

[4] E. H. Attia, "Phase synchronizing large antenna arrays using the spatial correlation properties of radar clutter," in Nat. Radio Sci. Meet. (URSI) Dig., 1984 p. 105.

[5] Y. Rockah and P. M. Schultheiss, "Array shape calibration using sources in unknown locations-Part I: Far-field sources," IEEE Trans. Acoust., Speech, Signal Processing, vol. ASSP-35, pp. 286-299, Mar. 1987.

[6] , "Array shape calibration using sources in unknown locations-Part II: Near-field sources and estimator implementation," IEEE Trans. Acoust., Speech, Signal Processing, vol. ASSP-35, pp. 724-735, Jun. 1987.

[7] B. Friedlander and A. J. Weiss, "Eigenstructure methods for direction finding with sensor gain and phase uncertainties," in Proc. Int Conf. Acoust., Speech, Signal Processing, New York, May 1988, pp. 2681-2684.

[8] J. T. Lo, "New maximum likelihood approach to multiple signal estimation," in Proc. Int. Conf. Acoust., Speech, Signal Processing, New York, May 1988, pp. 2889-2892.

[9] A. J. Weiss and B. Friedlander, "Array shape calibration using eigenstructure methods," in Proc. 23rd Asilomar Conf. Signals Syst. Comput., CA, Aug. 1989, pp. 925-929.

[10] D. A. Gray, W. O. Wolfe and J. L. Riley, "An eigenvector method for estimating the positions of the elements of an array of receivers," in Proc. Australian Symp. Signal Processing Applicat., Gold Coast, Australia, Aug. 1989, pp. 391-393.

[11] A. J. Weiss and B. Friedlander, "Array shape calibration using sources in unknown locations-A maximum likelihood approach," IEEE Trans. Acoust., Speech, Signal Processing, vol. 37, pp. 1958-1966, Dec. 1989.

[12] E. H. Attia and B. Steinberg, "Self-cohering large antenna arrays using the spatial correlation properties of radar clutter," IEEE Trans. Antennas Propagat., vol. 37, pp. 30-38, Jan. 1989.

[13] B. Friedlander and A. J. Weiss, "Direction finding in the presence of mutual coupling," IEEE Trans. Antennas Propagat., vol. 39, pp. 273-284, Mar. 1991.

[14] J. Pierre and M. Kaveh, "Experimental performance of calibration and direction finding algorithms," in Proc. Int. Conf. Acoust., Speech, Signal Processing, Toronto, Canada, May 1991, pp. 1365-1368.

[15] G. C. Brown, J. H. Clellan, and E. J. Holder, "Eigenstructure approach for array processing and calibration with general phase and gain perturbations," in Proc. Int. Conf. Acoust., Speech, Signal Processing, Toronto, Canada, June 1991, pp. 3037-3040.

[16] B. D. Steinberg and H. Subbaram, Microwave Imaging Techniques. New York: Wiley, 1991.

[17] M. Zhang and Z. Zhu, "Compensation for unknown mutual coupling in bearing estimation," Int. J. Electron., vol. 75, pp. 965-971, 1993.

[18] E. K. L. Hung, "Array element gain and phase estimation in the presence of interference," Defence Res. Establishment, Ottawa, Canada, Tech. Rep. 1211, Dec. 1993.
[19] E. H. Attia, "Statistics of the power pattern of a periodic array selfcohered using the SCA," in 11th Anпи. Benjamin Franklin Symp. Antenna Microwave Technol. 1990's-Symp. Dig., 1993, pp. 108-111.

[20] E. K. L. Hung, "Computation of the coupling matrix among the elements of an array antenna," in Proc. Int. Conf. Radar, Paris, France, July 1994, pp. 703-706.

[21] C. M. S. See, "Sensor array calibration in the presence of mutual coupling and unknown sensor gains and phases," Electron. Lett., vol. 30, pp. 373-374, Mar. 1994.

[22] _ "A method for array calibration in parametric sensor array processing," in Proc. Int. Conf. Communicat. Syst., Singapore, Sept. 1994, pp. 915-919.

[23] _ "Method for array calibration in high-resolution sensor array processing," Inst. Elect. Eng. Proc. Radar, Sonar, Navig., vol. 142, pp. 90-96, June 1995.

[24] M. Zhang and Z. Zhu, "Direction finding with unknown array sensor gain and phases," Int. J. Electron., vol. 78, pp. 199-207, 1995.

[25] _ "A method for direction finding under sensor gain and phase uncertainties," IEEE Trans. Antennas Propagat., vol. 43, pp. 880-883, Aug. 1995.

[26] B. C. Ng and A. Nehorai, "Active array sensor localization," Signal Processing, vol. 44, pp. 309-327, 1995.

[27] B. C. Ng and C. M. S. See, "Sensor-array calibration using a maximumlikelihood approach," IEEE Trans. Antennas Propagat., vol. 44, pp. 827-835, June 1996.

[28] I. S. D. Solomon, D. A. Gray, Yu. I. Abramovich, and S. J. Anderson, "Estimating of array mutual coupling and sensor positions for overthe-horizon radar," in Proc. Digital Signal Processing Applicat. Conf. (TENCON), Perth, Australia, Nov. 1996, pp. 846-851.

[29] _ "Over-the-horizon radar array calibration using echoes from ionized meteor trails," Inst. Elect. Eng. Proc. Radar, Sonar, Navigat., vol. 145, pp. 173-180, June 1998.

[30] I. S. D. Solomon, Y. I. Abramovich, D. A. Gray, and S. J. Anderson, "OTH radar antenna array calibration analysis," in Proc. Int. Symp. Signal Processing Applicat. (ISSPA), Gold Coast, Australia, Aug. 1996, pp. 471-474.

[31] I. S. D. Solomon, D. A. Gray, Yu. I. Abramovich, and S. J. Anderson, "Sources for OTH radar array calibration," in Proc. IEEE Antennas Propagat. Symp., Montreal, Canada, July 1997, pp. 306-309.

[32] _ "Meteor echoes as sources of opportunity for over-the-horizon radar array calibration," Radio Sci., to be published.

[33] I. J. Gupta and A. A. Ksienski, "Effect of mutual coupling on the performance of adaptive arrays," IEEE Trans. Antennas Propagat., vol. AP-31, pp. 785-791, Sept. 1983.

[34] R. O. Schmidt, "A signal subspace approach to multiple emitter location and spectral estimation," Ph.D. dissertation, Stanford Univ., 1981.

[35] M. Zoltowski and F. Haber, "A vector space approach to direction finding in a coherent multipath environment," IEEE Trans. Antennas Propagat., vol. AP-34, pp. 1069-1079, Sept. 1986.

[36] I. S. D. Solomon, "Over-the-horizon radar array calibration," Ph.D. dissertation, Univ. Adelaide, Australia, Apr. 1998.

[37] M. Wax and T. Kailath, "Determining the number of sources impinging on a passive array by Akaike's information criterion," Inform. Syst. Lab., Stanford Univ., 1983.

[38] Yu. I. Abramovich, S. J. Anderson, and I. S. D. Solomon, "Adaptive ionospheric distortion correction techniques for HF skywave radar," in Proc. IEEE Nat. Radar Conf., Ann Arbor, MI, May 1996, pp. 267-272.

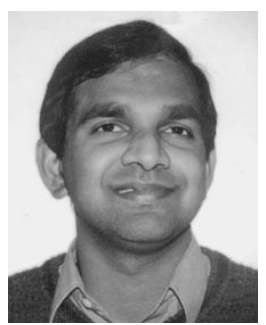

Ishan Samjeva Daniel Solomon (S'91-M'93) was born in Sri Lanka in 1971. He received the B.Eng. (honors) (electrical and electronic engineering), B.Sc. (mathematics and computer science), and Ph.D. degrees, all from the University of Adelaide, Australia, in 1992, 1993, and 1998, respectively.

In December 1993 he joined the Defence Science and Technology Organization, Adelaide, Australia, and has since been working on both skywave and surface wave over-the-horizon radars. His work has mainly involved the temporal and spatial processing of radar signals, but he has also worked on projects relating to ionospheric propagation of over-the-horizon radar signals. His research interests are in array processing, digital signal processing, ionospheric studies and radar systems. His most significant contribution was showing that radar echoes from meteor trails can be used to estimate unknown or imprecisely known parameters of a radar's antenna array. 


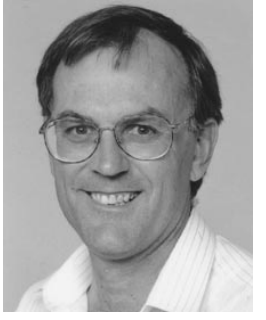

Douglas A. Gray (M'82) was born in the United Kingdom in 1946. He received the B.Sc. (honors) and $\mathrm{Ph} . \mathrm{D}$. degrees in mathematical physics from the University of Adelaide, South Australia, in 1969 and 1973, respectively.

From 1973 to 1993 he was with the Australian Defence Science and Technology Organization applying signal processing to sonar and electronic warfare. From 1977 to 1979 he was a Visiting Scientist at the Royal Aircraft Establishment, U.K., and in 1985 was a Visiting Fellow at the Australian National University, Canberra, Australia. He is currently a Professor of sensor signal processing at the University of Adelaide, South Australia, and Deputy Director of the Cooperative Research Centre for Sensor Signal and Information Processing, Adelaide, Australia. His research interests are in the application of signal processing to sonar, radar, global positioning systems and electronic warfare, particularly in adaptive processes, beamforming, signal sorting and classification techniques, data fusion, and association.

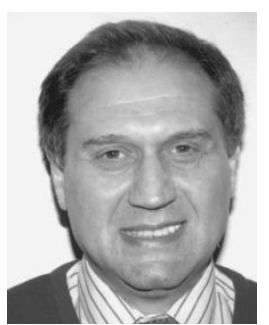

Yuri I. Abramovich (M'96) received the Dip.Eng. (honors) degree in radio electronics in 1967 and the Candidate of Science degree (Ph.D. equivalent) in theoretic radio techniques in 1971, both from the Odessa Polytechnic University, USSR, and the D.Sc. degree in radar and navigation from the Leningrad Institute for Avionics in 1981.

From 1968 to 1994, he was with the Odessa State Polytechnic University, Ukraine, as a Research Fellow, Professor, and finally as Vice-Chancellor of Science and Research. Since 1994 he has been at the Cooperative Research Centre for Sensor Signal and Information Processing, South Australia, as a Visiting Professor. His research interests are in the application of signal processing to radar, particularly over-the-horizon radar, electronic warfare and communication, particularly spatio-temporal adaptive processing, beamforming, signal detection, and estimation.

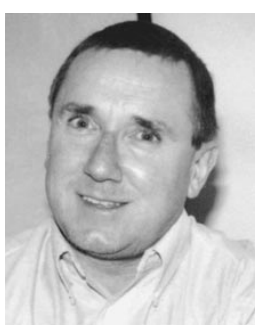

Stuart J. Anderson was born in Perth, Western Australia, on May 1, 1946. He received the B.Sc. and Ph.D. degrees in physics from the University of Western Australia, Perth, in 1968 and 1972, respectively.

Since 1974, he has been a member of the team which has developed the Jindalee over-the-horizon radar system, with responsibilities for ship detection, remote sensing and target classification. He spent 1986-1987 as a Visiting Scientist at the U.S. Naval Research Laboratory, Washington, DC, and from 1987 to 1988 in a similar position at the U.K. Admiralty Research Establishment. His current research embraces the application of adaptive signal processing techniques to the problem of ionospheric distortion correction, electromagnetic scattering from rough time-varying surfaces, HF radar polarimetry, nonlinear dynamics of ocean waves, and the design of HF radar systems for a wide variety of missions.

Dr. Anderson received the Minister of Defence Science Award for Research Achievement in 1992. 